\title{
El subregistro potencial de accidentes de trabajo en el Instituto Mexicano del Seguro Social
}

\author{
José Santiago Salinas-Tovar, MC, (1) Pablo López-Rojas, MC, M en C, ${ }^{(1)}$ \\ María $O$ ralia Soto-N avarro, MC, M en $C,{ }^{(1)} D$ avid Efraín C audillo-Araujo, MC, ${ }^{(1)}$ \\ Francisco Raúl Sánchez-Román, MC, M en C, ${ }^{(1)}$ Víctor Hugo Borja-Aburto, MC , PhD. ${ }^{(1)}$
}

\section{Salinas-Tovar JS, López-Rojas P, Soto-Navarro MO, Caudillo-Araujo DE, Sánchez-Román FR, Borja-AburtoVH. El subregistro potencial de accidentes de trabajo en el Instituto Mexicano del Seguro Social. Salud Publica Mex 2004;46:204-209. El texto completo en inglés de este artículo está disponible en: http://www.insp.mx/salud/index.html}

\section{Resumen}

Objetivo. Evaluar el potencial subregistro de casos de accidentes de trabajo atendidos en el Instituto Mexicano del Seguro Social. Material y métodos Estudio transversal Ilevado a cabo en México con información a escala nacional proveniente de 27 Delegaciones del Instituto Mexicano del Seguro Social (IMSS) sobre trabajadores atendidos por probable accidente de trabajo, segun declaración del trabajador en su primera atención en los servicios de urgencia de hospitales del IMSS durante el mes de noviembre de 2001, que posteriormente se comparó con los registros de casos reclamados o reconocidos oficialmente como accidentes de trabajo, lo que permitió identificar la proporción de los mismos que no terminaron el proceso de calificación. El análisis de la información se hizo a través de estadística descriptiva para cada variable; la estimación anual de las proporciones encontradas se realizó mediante la multiplicación por 12 meses y los intervalos de confianza se estimaron en $95 \%$ con el método exacto de Poisson para una proporción. Resultados. La información de 27 de $37 \mathrm{De}$ legaciones del IMSS reveló que 7211 casos no fueron reconocidos como accidentes de trabajo en el periodo de estudio; lo que equivaldría a un subregistro de accidentes de trabajo nacional de $26.3 \%$, con variaciones en las D elegaciones de 0 a 68\%. Los diagnósticos más frecuentes fueron contusiones y heridas leves. Conclusiones. El subregistro encontrado puede afectar las prestaciones a trabajadores y
Salinas-Tovar JS, López-Rojas P, Soto-Navarro MO, Caudillo-Araujo DE, Sánchez-Román FR, Borja-AburtoVH. Potential under-registration of occupational accidents in the Mexican Institute of Social Security.

Salud Publica Mex 2004;46:204-209.

The English version of this paper

is available at: http://www.insp. mx/salud/index.html

\begin{abstract}
A bstract
Objective. To assess the potential under-registration of work-related accidents in the Mexican Institute of Social Security. Material and Methods. A countrywide crosssectional study was carried out with information collected from 27 district offices of the Mexican Institute of Social Security (MISS), on workers seen at MISS emergency rooms during N ovember 2001 because of a probable accident at work. We compared these reports to official records of work-related accidents to estimate the proportion of incomplete reports. D ata analysis consisted of descriptive statistics for each variable; the annual estimation of inco mplete reporting proportions was made by multiplying by twelve months; 95\% confidence intervals were estimated using Poisson's exact method for a proportion. Results $D$ ata from 27 out of 37 MISS district offices revealed that 7211 cases were not recognized as work accidents, accounting for an underestimation of $26.3 \%$, ranging between 0 and $68 \%$ among the different district offices. The accidents that were most frequently left unregistered were mild and blunt injuries. Conclusions Under-registration can affect worker compensation plans and the financial balance of the institution's occupational risk insurance. Research is needed to investigate and eliminate the causes of under-registration. Employers, the industry, and health institutions should be involved in this effort.The English version of this paper is available at: http://www.insp.mx/salud/index.html
\end{abstract}

(1) Coordinación de Salud en el Trabajo, Instituto Mexicano del Seguro Social, México, DF, México.

Fecha de recibido: 12 de mayo de 2003 - Fecha de aprobado: 20 de febrero de 2004

Solicitud de sobretiros: Dr. José Santiago Salinas Tovar. Avenida Cuauhtémoc 330, Edificio C , 30 piso, colonia D octores, Delegación Cuauhtémoc, 06725 México, DF, México

Correo electrónico: santiago.salinas@ imss.gob.mx 
el equilibrio financiero del seguro de riesgos de trabajo. Sin embargo, to davía es necesario investigar sus causas y los factores para abatirlo, debiendo participar empresarios, instituciones de trabajo y de salud. El texto completo en inglés de este artículo está disponible en: http://www.insp.mx/salud/index.html

Palabras clave: accidentes de trabajo; incidencia; registros médicos; seguridad social; México
Key words: accidents, occupational; work-related accident registry; incidence; medical records; so cial security; M exico
E 1 registro de los accidentes y enfermedades profesionales ha sido reconocido como un problema en la mayor parte de los países, debido a deficiencias en los sistemas de información e insuficiente coordinación entre los diferentes organismos de trabajo y salud. De este problema no están exentos los países desarrollados; la Oficina de Estadísticas de Trabajo de Estados Unidos de América (EUA) reporta que del total de accidentes y enfermedades, aproximadamente $10 \%$ no son registrados y los días de trabajo perdidos por lesiones y enfermedades de estos casos son aproximadamente $25 \%{ }^{1,2}$

Una de las causas de subregistro es el escaso reconocimiento de la importancia de la problemática por parte de empresas y trabajadores, ${ }^{3-5}$ especialmente en las enfermedades de trabajo, que por su naturaleza requieren de un tiempo prolongado para la presentación de signos y síntomas. ${ }^{6,7}$ En un estudio realizado por Robin ${ }^{8}$ sobre las defunciones por enfermedades relacionadas con el trabajo, señala que éstas son 10 veces más que las generadas por accidentes de trabajo (en EUA se reporta una tasa de 5.3 por cada 100000 habitantes).

En el Instituto Mexicano del Seguro Social (IMSS), el seguro de riesgos de trabajo (que incluye accidentes y enfermedades) garantiza la atención médica, el pago de días de incapacidad temporal que contempla 100\% del salario de cotización desde el primer día del siniestro hasta por 365 días, dependiendo de la naturaleza de la lesión, y en caso de sufrir alguna pérdida orgánica o funcional otorga la indemnización correspondiente de acuerdo con la Ley Federal del Trabajo, ya sea en forma de pago global o pensión., ${ }^{9,10}$

Las cuotas que por el seguro de riesgos de trabajo deben pagar los patrones se determinan en relación con la cuantía del salario y con las consecuencias de los riesgos de trabajo de sus trabajadores, como los días de incapacidad temporal para el trabajo, las incapacidades permanentes y las defunciones. ${ }^{11}$

Durante las últimas décadas se ha observado un descenso en la frecuencia absoluta y relativa de los ries- gos de trabajo; durante 1980 se registraron 598677 accidentes de trabajo (incluidos los accidentes en trayecto) entre los 5077948 de trabajadores inscritos en este ramo de seguro; en 2001 se registraron 408228 de accidentes entre los 12224231 trabajadores inscritos. Así, la tasa de riesgos de trabajo en las empresas afiliadas al IMSS pasó de 11.8 en 1980 a 3.4 por cada 100 trabajadores en 2001 (figura 1). ${ }^{12}$ Las razones de esta disminución no han sido bien documentadas, pero pueden atribuirse a múltiples causas: desde la modificación de los procesos de trabajo y el desarrollo tecnológico que ha permitido la automatización de trabajos riesgosos, mayor conocimiento por parte de empresas y sindicatos sobre los riesgos potenciales en los lugares de trabajo, hasta la implantación de programas de salud y seguridad, con estímulos para la prevención. Otros factores, que podrían influir en un menor reporte y registro de éstos han sido establecidos por Montes de Oca, ${ }^{13}$ quien encontró en una encuesta aplicada a trabajadores lesionados que 53\% desconocía el procedimiento de reclamación, 26\% no reclamaron el accidente por considerar un trámite burocrático y 22\% desconocía que era un accidente de trabajo.

De especial interés para el IMSS es el subregistro, ya que por un lado se ven afectados los asegurados, al no obtener las prestaciones a que tienen derecho y verse obligados a obtener beneficios de otro tipo de seguro, como lo es el seguro de enfermedad general, en donde destaca el pago de incapacidades temporales para el trabajo con $60 \%$ del salario de cotización, a partir del cuarto día, el no tener derecho al otorgamiento de prótesis y órtesis; en caso de presentar secuelas, éstas no son indemnizadas y no contar con el derecho a la reincorporación laboral al resolverse su patología. Por otra parte, se afecta al IMSS al dejar de percibir las cuotas reales correspondientes al seguro de riesgos de trabajo, que es pagado en su totalidad por los empresarios.

El proceso para la calificación o certificación de los riesgos de trabajo en el régimen del IMSS establece que cuando un trabajador sufre una lesión al desem- 


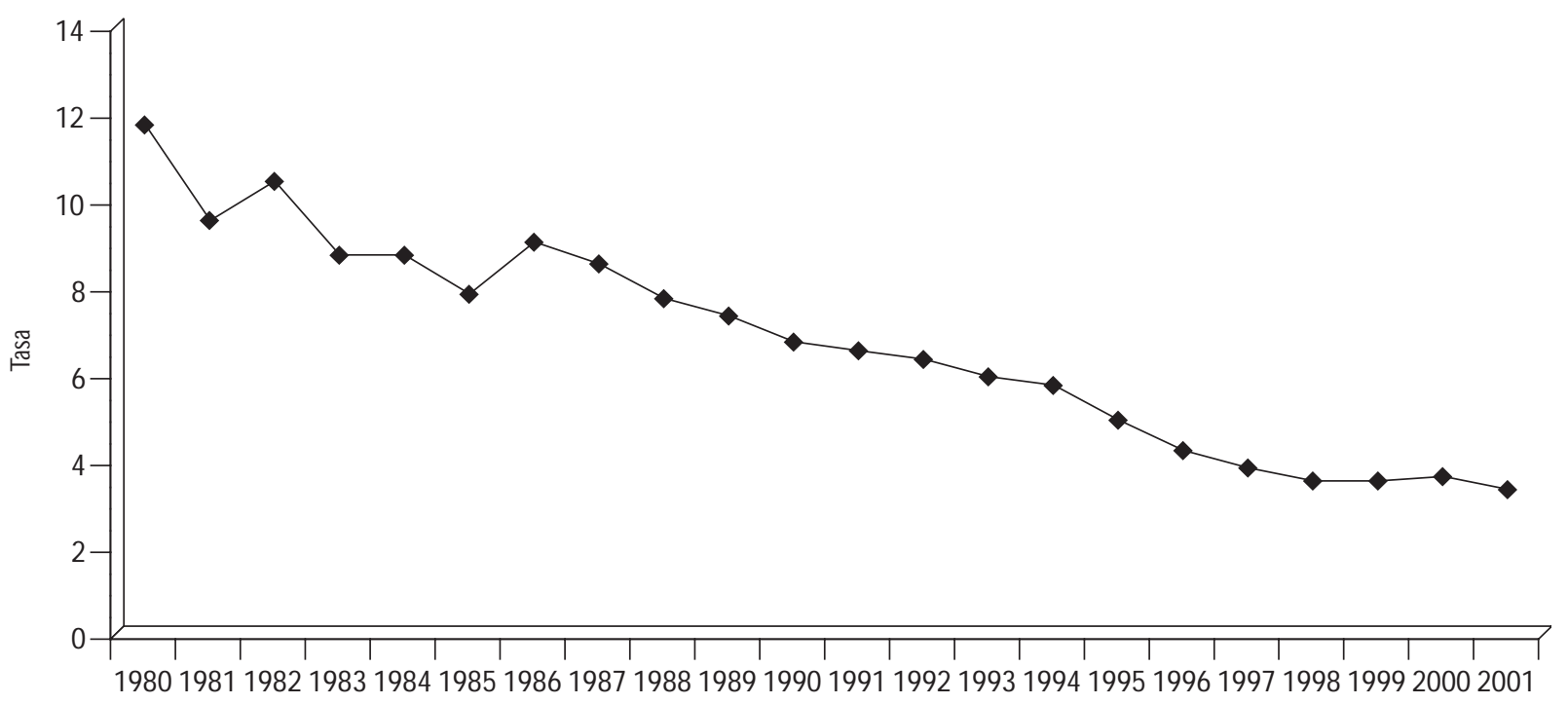

Figura 1. Tasa de riesgos de trabajo por cada 100 trabajadores de 1980 a 2001. Instituto Mexicano del Seguro Social, 1980-2001

peñar sus actividades laborales, o con motivo de éstas, debe acudir para atención médica a los servicios institucionales en los cuales, una vez atendido, se registra en un formato especial como probable riesgo de trabajo y se le debe proporcionar el "formato o aviso para calificar probable riesgo de trabajo" (ST-1), que deberá requisitar el patrón para que, posteriormente, los Servicios de Salud en el Trabajo dictaminen si es o no considerado como riesgo de trabajo, para que se otorguen las prestaciones de este ramo de seguro. El trámite mencionado requiere entonces de la gestión activa del trabajador o su familia y de la descripción del accidente por parte de la empresa; si alguno de éstos no se realiza, no se concluye con el proceso de calificación ni con el registro del accidente. ${ }^{14}$

Para dimensionar la magnitud del problema se desarrolló el presente trabajo, con el que se busca identificar el grado de subregistro de accidentes de trabajo en trabajadores que acuden inicialmente a atención médica institucional como consecuencia de un probable accidente de trabajo.

\section{Material y métodos}

Estudio transversal llevado a cabo en noviembre de 2001, para analizar la información proveniente de los servicios de urgencias de 27 Delegaciones del IMSS, relacionada con la atención de primera vez de trabajadores que consultaron por accidente de trabajo. Se revisó el comportamiento mensual de los riesgos de trabajo de 2001; como la mediana se ubicó en el mes de noviembre, se seleccionaron los casos de este mes que acudieron para atención médica a los servicios de urgencias y medicina familiar en los cuales se identificó que el padecimiento había sido generado al estar desempeñando sus actividades laborales o con motivo de éstas. Se llenó el formato de "atención de un probable riesgo de trabajo" (ST-4-30-8), sin concluir el trámite para calificación por riesgo de trabajo. Se recolectaron todos los formatos ST-4-30-8 del mes de referencia que no prosiguieron con el trámite médico- administrativo en los Servicios de Salud en el Trabajo.

La información contenida en los formatos ST-430-8 contempla unidad médica, Delegación, número de seguridad social, edad, sexo, fecha del accidente, diagnóstico y los días iniciales de incapacidad temporal otorgados para el trabajo. Se identificó en la base de datos del sistema de registro nacional de riesgos de trabajo ocurridos y terminados del IMSS si estos casos habían sido calificados como accidentes de trabajo en cualquiera de las Delegaciones del sistema y de ser así fueron eliminados.

En una muestra de los trabajadores que reportaron haber sufrido un accidente de trabajo y que no terminaron el proceso de calificación, se investigó si se encontraban dados de alta por sus empresas ante el IMSS al momento del accidente, así como si se les había otorgado incapacidad temporal para el trabajo por 
este padecimiento, y bajo qué ramo de seguro se les otorgó. Para determinar el tamaño de la muestra se consideró una prevalencia de $20 \%$ de casos no vigentes, con un intervalo de confianza de $95 \%$ y un error de $5 \%$.

La estimación anual de las proporciones encontradas se realizó mediante la multiplicación por 12 meses, y los intervalos de confianza se estimaron a 95\% con el método exacto de Poisson para una proporción.

\section{Resultados}

Se recabó información de 27 de las 37 Delegaciones del sistema, con un total de 7211 casos. En las Delegaciones 3 Suroeste y 4 Sureste del Distrito Federal, Aguascalientes, Coahuila, Nuevo León, San Luis Potosí, Baja California, Nayarit, Puebla y Veracruz Sur no fue posible obtener la información debido a un manejo distinto de los formatos requeridos (cuadro I).

Se identificaron 7211 casos atendidos por probable riesgo de trabajo que no prosiguieron el trámite de calificación de profesionalidad del riesgo. De éstos, $85.2 \%$ fueron probables accidentes de trabajo y $14.8 \%$, accidentes en trayecto; $74 \%$ correspondieron al sexo masculino y $26 \%$ al sexo femenino, con un promedio de edad de 29.6 y 32.8 años, respectivamente.

El número de casos de accidentes que sí se registró porque concluyeron su trámite en el mes de noviembre en las Delegaciones estudiadas fue 20 207, por lo que el subregistro promedio encontrado fue de $26.3 \%$, con un rango de 0 a $68 \%$ por Delegación. El $59.2 \%$ de las Delegaciones tuvieron un subregistro superior a 30\%. De las cuatro Regiones en las que se agrupan las Delegaciones, la Región Sur presenta el dato más alto con
$35.4 \%$ y casi la totalidad de sus Delegaciones están por arriba de 20\%; la Región Centro tiene el menor subregistro, 15.7\% (cuadro I).

De esta manera el número estimado de casos no registrado en el año 2001 es de 85251 (IC 95\% 82 982-87 196). Los diagnósticos más frecuentes fueron contusiones con $24 \%$ para hombres y $34 \%$ para las mujeres, en segundo lugar heridas y esguinces y en tercero dorsalgias y lumbalgias, respectivamente, lesiones consideradas leves (cuadro II).

En menor proporción se identificaron casos de fracturas de diferentes partes del cuerpo, así como amputaciones de dedos de mano, lesiones graves que tardan en sanar más de 15 días y que pueden generar secuelas permanentes.

Fue posible reconocer empresas que presentan hasta 10 casos de trabajadores con probable accidente de trabajo no reclamados en el mes de estudio, lo que indica una conducta inadecuada, ya que se les proporcionó la atención médica inicial que es la más costosa y únicamente continuaron su control médico fuera del IMSS.

La muestra en la que se investigó la vigencia y el otorgamiento de incapacidades de los casos no registrados fue de 806 trabajadores; de éstos, 6\% no se encontraban dados de alta en el seguro de riesgos de trabajo al momento de la lesión, lo que constituye una violación a las disposiciones de la Ley del Seguro Social sujetas a la aplicación de severas sanciones económicas. De los 806 trabajadores, 424 casos gozaron de al menos un día de incapacidad temporal para el trabajo. El promedio de días por caso fue de 6 con un rango de 2 a 29 días, lo que totalizó en la muestra 2748 días de incapacidad, que para el total de casos no regis-

\section{Cuadro I

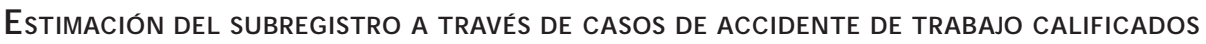 Y los no reclamados para calificación en Noviembre 2001, por Delegaciones y Regiones del Instituto Mexicano del Seguro Social}

\begin{tabular}{|c|c|c|c|c|c|c|}
\hline Región & $\begin{array}{l}\text { Accidentes de trabajo, } \\
\text { reclamados y calificados } \\
\text { noviembre } 2001^{*}\end{array}$ & $\begin{array}{c}\text { Probables accidentes } \\
\text { de trabajo, no reclamados } \\
\text { noviembre } 2001^{*}\end{array}$ & Total & $\begin{array}{c}\% \\
\text { Subregistro }\end{array}$ & $\begin{array}{c}\text { Tasa anual } 2001 \\
\text { de accidentes } \\
\text { de trabajo observada } \\
\text { por } 100 \text { trabajadores* }\end{array}$ & $\begin{array}{l}\text { Tasa anual } 2001 \text { de accidentes } \\
\text { de trabajo corregida } \\
\text { por subregistro } \\
\text { por } 100 \text { trabajadores* }\end{array}$ \\
\hline Centro (7 D elegaciones) & 7823 & 1458 & 9281 & 15.7 & 3.56 & 4.12 \\
\hline N orte (4 D elegaciones) & 2826 & 792 & 3618 & 21.9 & 2.66 & 3.24 \\
\hline 0 ccidente (7 Delegaciones) & 6936 & 3523 & 10459 & 33.7 & 3.68 & 4.92 \\
\hline Sur (9 D elegaciones) & 2622 & 1438 & 4060 & 35.4 & 2.50 & 3.39 \\
\hline N acional (27 D elegaciones) & 20207 & 7211 & 27418 & 26.3 & 3.3 & 4.11 \\
\hline
\end{tabular}

Fuente: casos no reclamados y Memoria Estadística N acional de Riesgos de Trabajo, Instituto Mexicano del Seguro Social, 2001 


\section{Cuadro II \\ DISTRIBUCIÓN, POR SEXO, DE LOS PRINCIPALES DIAGNÓSTICOS DE ACCIDENTE DE TRABAJO No Reclamados en MéXico, NOVIEMBRe 2001}

\begin{tabular}{lrrlrr} 
Sexo masculino & No. & $\%$ & Sexo femenino & No. & $\%$ \\
1. Contusiones & 1281 & 24 & 1. Contusiones & 638 & 34 \\
\hline 2. Heridas & 960 & 18 & 2. Esguinces & 394 & 21 \\
\hline 3. D orsalgias & 587 & 11 & 3. Lumbalgias & 187 & 10 \\
\hline 4. Cuerpo extraño en ojo & 480 & 9 & 4. Heridas & 131 & 7 \\
\hline 5. Lumbalgias & 373 & 7 & 5. Q uemaduras & 75 & 4 \\
\hline 6. 0 tros & 1655 & 31 & 6. 0 tros & 450 & 24
\end{tabular}

Fuente: Base de datos de casos no reclamados como accidentes de trabajo en el ámbito nacional del Instituto Mexicano del Seguro Social

trados en el mes de noviembre correspondería a 46 150, y, anualmente, a 553804 días de incapacidad otorgados en la rama de enfermedad general, cuando realmente corresponden a accidentes de trabajo.

\section{Discusión}

Las estadísticas oficiales del IMSS muestran una tasa de riesgos de trabajo en la población trabajadora asegurada muy por debajo de lo reportado en otros países desarrollados. En Francia la tasa pasó de 4.63 accidentes de trabajo por cada 100 trabajadores en 1995 a 4.43 en 1999; en Dinamarca, de 1.92 a 1.8 en el mismo periodo; en Canadá de 3.64 a 3.14; aun considerando que las condiciones de seguridad e higiene de las empresas de estos países son mejores que las nuestras, 15,16 lo cual hace suponer un subregistro de accidentes de trabajo en los trabajadores afiliados al Instituto Mexicano del Seguro Social. Macías ${ }^{17}$ identificó un subregistro de accidentes de trabajo de 53\% en Nuevo León; Jaramillo ${ }^{18} 48 \%$ en el estado de Baja California; similares resultados obtuvo Franco ${ }^{19}$ en una empresa textil del Estado de México.

El porcentaje de subregistro que se identificó en este estudio en el ámbito nacional contempla únicamente los casos atendidos por los servicios médicos del Instituto; faltaría investigar la existencia de casos que son atendidos por servicios médicos privados o de las propias empresas que no son informados al Instituto como accidentes de trabajo, lo que incrementaría la cifra del subregistro, como lo demuestra el estudio realizado por Colunga, ${ }^{20}$ en el cual identificó que 16\% de los trabajadores que sufrieron un accidente de trabajo no estaban dados de alta en el IMSS, lo que puede generar que un número de trabajadores no afiliados sean atendidos en servicios médicos ajenos al IMSS, con el fin de no ser sancionados; además, se observó que el personal médico de las empresas conocía parcialmente el manejo médico administrativo que debía seguir.

Por otra parte, habría que reconocer como una posible causa de subregistro el procedimiento administrativo para la calificación de riegos de trabajo, que por sí mismo podría explicar los casos que generaron por lo menos un día de incapacidad y no continuaron el trámite, por lo que su simplificación o adecuación debe plantearse.

En los casos estudiados fue posible identificar la existencia de diagnósticos que no sólo generan días de incapacidad temporal para el trabajo, sino también secuelas permanentes que deterioran el nivel de salud de la población trabajadora.

Dada la magnitud de este problema es necesario continuar con líneas de investigación que permitan identificar con mayor precisión los factores que generan el subregistro de los accidentes de trabajo y su peso específico, para mejorar la calidad de las estadísticas y, por ende, optimizar la atención de los trabajadores, otorgándoles las prestaciones a las que tienen derecho, así como apoyar a las empresas en el mejoramiento de las condiciones de seguridad e higiene.

\section{Agradecimientos}

Nuestro reconocimiento, por su participación en las reuniones del diagnóstico sobre el registro de riesgos de trabajo, a: Dr. Alejandro Guarneros Chumacero, C.P. Mario Alberto Rosas Rello, Lic. Manuel González Sotomayor, Lic. Rosa María Sandoval García, Lic. Marina Araceli Castro Moreno, Lic. Luis Díaz Reyes, Lic. Sergio Gatica Suárez, Lic. Luis David Ortega Ramírez, Lic. Eduardo Escalante Macín, Mat. Héctor Argüelles Tejeda, Lic. Teresa Romero. Sra. Martha Matilde Valle Soto, Dra. Yolanda Guerra Márquez.

\section{Referencias}

1. $O$ ccupational Safety and $\mathrm{Health} A$ dministration. Department of Labour.Yearbook statistics of work. W ashington, DC: O SHA; 2000: 28-32.

2. 0 ccupational Safety and $\mathrm{H}$ ealth Administration. D epartment of Labour. W orkplace injury, illness and fataly statistics, 1996.W ashington, DC: OSHA.

3.W erner AF. 0 ccupational health in Argentina.Arch 0 ccup Environ Health 2000;73:285-289.

4. Fengsheng $\mathrm{HE}$. $\mathrm{O}$ ccupational medicine in China. Arch $\mathrm{O}$ ccup Environ Health 1998;71:79-84.

5. Koh JD. 0 ccupational health in Singapore. Arch 0 ccup Environ Health 1998;71:295-301. 
6. $O$ leinick A, G uire KE, Howtorne VM, Schork MA, Cluck US, Lee BH. $C$ urrent methods of estimating severity for occupational injuries and illnesses: D ata from the 1986 Michigan comprehensive compensable injury and illness data base.Am J Ind Med 1993;23:231-252.

7. Murphy LP, Sorok SG, Courtney KT, W ebster SB, Leamon BI. Injury and illness in the American workplace:A comparison of data sources. Am J Ind Med 1996; 30:130-141.

8. Robin H, Landringan PJ.W ork-related death:A continuing epidemic. Am J Public Health 2000;90:541-545.

9. Instituto Mexicano del Seguro Social. Ley del Seguro Social. México, DF: Coordinación G eneral de Comunicación Social, IMSS;2001.

10. Breña G F. Ley Federal del Trabajo comentada y concordada. $2^{\circ}$ edición. México, DF:Editorial Harla; 1988.

11. Instituto Mexicano del Seguro Social. Reglamento para la Clasificación de Empresas y D eterminación de la Prima en el Seguro de Riesgos de Trabajo. México, DF: Coordinación General de Comunicación Social, IMSS; 2001.

12. Instituto Mexicano del Seguro Social. Memoria Estadística Institucional IMSS 1986-2001. México, DF:IMSS; 2002.

13. Montes de 0 ca M. Subregistro de riesgos de trabajo en el Estado de México.V Reunión N acional de Investigación en Salud en el Trabajo Memorias. México, DF:IMSS;2001.
14. Colunga GM. Servicios Médicos en empresas de Cancún, con alto índice de accidentes de trabajo. Rev Med Inst Mex Seguro Soc 1993;31:395-399.

15. Conway H, Svenson J. $O$ ccupational injury and illness rates, 1992 1996:W hy they fell?. Monthly Labor Rev 1998;121:36-59.

16.Van-C harante AW, Mulder PG. Reporting of industrial accidents in the N etherlands.Am J Epidemiol 1998;148:182-190.

17. Macías RE, Pérez PA. Seguimiento administrativo de los probables riesgos de trabajo atendidos en el servicio de urgencias adultos del H.G .R.46 Guadalajara Jalisco. III Reunión N acional de Investigación en Salud en elTrabajo Memorias. México, DF; IMSS; 1999.

18. Jaramillo MA. Factores que influyen en la no reclamación de los riesgos de trabajo. II Reunión N acional de Investigación en Salud en el Trabajo. Memorias. México, DF: 1998

19. Franco E, Cuéllar R, N oriega E, Villegas R. Los trabajadores textiles de N aucalpan, Estado de México y sus condiciones de salud en 1989. Salud Problema 1994;invierno; (24)33-43.

20. Colunga $G, C$ ervantes $R$, Fuentes $R$. Inconcluso trámite administrativo del reclamo de riesgos de trabajo. II Reunión Nacional de Investigación en Salud en el Trabajo. Memoria México, DF: IMSS; 1998; Resumen 41:76. 